\title{
CFD study of Particle Flow Patterns in a Rotating Cylinder Applying OpenFOAM and Fluent
}

\author{
Susantha Dissanayake $^{1} \quad$ Sumudu S Karunarathne $^{1} \quad$ Joachim Lundberg $^{1} \quad$ Lars-Andre Tokheim $^{1}$ \\ ${ }^{1}$ Department of Process, Energy, and Environmental Technology, University College of Southeast Norway, Norway. \\ susanthadmscdegmail.com, \{Sumudu.Karunarathne, Joachim.Lundberg, \\ Lars.A.Tokheim\} Qusn. no
}

\begin{abstract}
A rotating cylinder $(\mathrm{RC})$ is a common type of reactor used in the industry, the most typical example being a cement kiln. The particle flow pattern inside such a unit is necessary for the mass and energy transfer, and this flow pattern depends on the operational Froude number and the degree of filling. The main aim of this study is to compare the simulation results from OpenFOAM and Fluent applying a Eulerian multiphase flow modeling concept to study the behavior of dense particle gas mixtures under different operational conditions. Six different flow patterns are simulated, varying the degree of filling from 10 to $45 \%$ and the Froude number from 0.0001 to 5 . OpenFOAM is capable of producing results very close to those generated with Fluent, and both software appears to be suitable for simulating the RC dense particle flow using the Eulerian approach.
\end{abstract}

Keywords: Particle flow, OpenFOAM, Fluent, Rotating cylinder, Froude number

\section{Introduction}

The behavior of particles inside a rotating cylinder (RC) has a significant influence on the mass and energy transfer, both in the solids phase and between the solids and the surrounding medium [1-4]. A cement kiln is a typical example of a $\mathrm{RC}$ which has mass and energy transfer between the phases [5, 6]. Six different flow patterns can be identified, depending on the degree of filling and the rotational speed; slipping, slumping, rolling, cascading, cataracting and centrifuging $[2,7]$.

Such flow patterns have previously been simulated by some of the authors using the CFD software Fluent, applying the Eulerian approach to model both the particle phase and the gas phase [8].

OpenFOAM is an open source CFD toolbox that can be downloaded and used free of charge ${ }^{1}$. The OpenFOAM solver "twoPhaseEulerFoam" can be used to model incompressible fluid phases, such as one particle phases and one gas phase. This approach applies the Eulerian conservation equations for both the gas and the solids phases, meaning that the phases are modeled as interpenetrating continua $[2,9,10]$.
The purpose of the current study is to investigate the ability of OpenFOAM to map the flow pattern mentioned above and to compare the simulation results with those from Fluent simulations.

\section{Theoretical background}

Heydenrych [7] studied the flow pattern inside a RC for particles in size range 0.1-10 $\mu \mathrm{m}$. The flow pattern depends on the degree of filling and the Froude number $(F r)$. The degree of filling can be expressed as the ratio $H / R$, i.e. bed depth $H(m)$ to cylinder radius $R(m)$. The Froude number is a function of the rotational speed $\omega$ $\left(\frac{\mathrm{rad}}{\mathrm{s}}\right)$, the cylinder radius and the gravitational constant, $g\left(9.81 \frac{\mathrm{m}}{\mathrm{s}^{2}}\right)$ :

$$
F r=\frac{\omega^{2} R}{g}
$$

Six different flow patterns can be mapped into the $\mathrm{Fr}-\mathrm{H} / \mathrm{R}$ space based on the particle behaviour: Slipping slumping, rolling, cascading, cataracting and centrifuging [7, 8], see Figure 1.

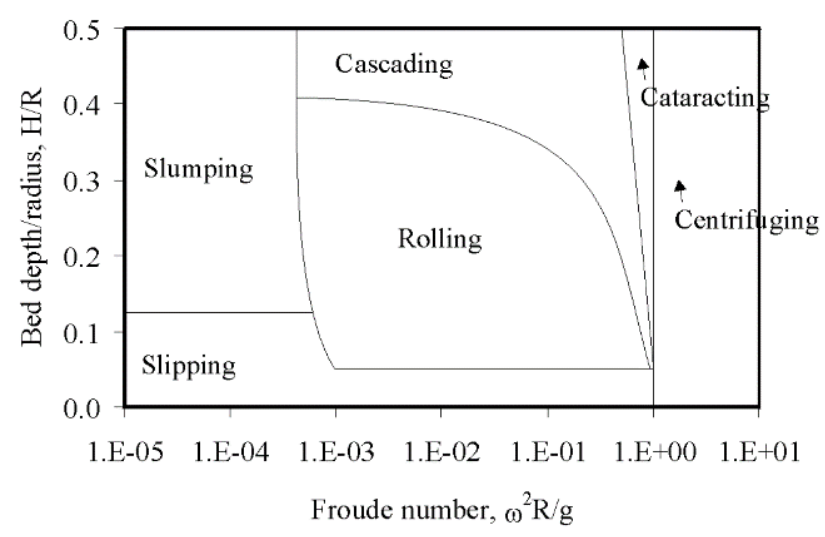

Figure 1. Flow regime as a function of operational conditions [7].

A particle bed in motion may have an active and a passive layer. When the cylinder rotates, the particles adjacent to the wall obtain some velocity. Other particles are located at a distance from the cylindrical wall and stay stagnant $[2,7,11,12]$. The particle layer

\footnotetext{
${ }^{1}$ www.openfoam.com
} 
that gains some velocity due to the cylindrical wall rotation is called the active layer, whereas the stagnant layer of particles is referred to as a passive layer. When the rotational speed increases, the active layer thickness increases. An illustration of active and passive layers is shown in Figure 2.

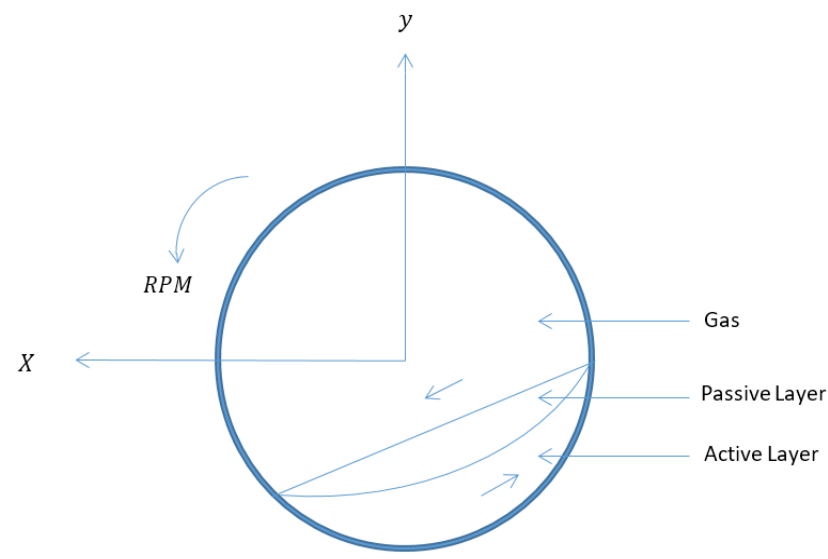

Figure 1. The active and passive layers development in an RC.

\subsection{Basic Flow patterns}

The six different basic flow patterns are described in this section.

\subsubsection{Slipping}

The slipping mode can be observed for $H / R<0.15$ and $F r<0.001$. In the slipping mode, the active layer shows very little particle movement [7, 13], and the active layer is very thin compared to the passive layer, see Figure 3. In other words, the passive layer is predominant in this flow mode. As a result, the particle mixing, the mass trasfers and the heat transfer between paticles and between gas and particles is at a minimum.

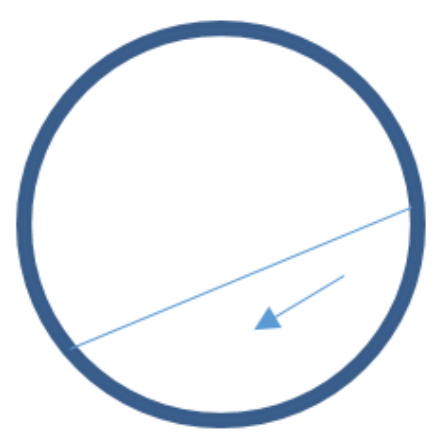

Figure 3. The slipping mode.

\subsubsection{Slumping}

If $F r$ is kept below 0.001 , but $H / R$ is increased above 0.15 , the particle bed tends to move with the moving wall, but then at a some elevation the particles suddenly collapse and move back towards the initial position [13], see Figure 4. Due to the slumping behavior, the bed is predominantly a passive layer, hence slumping is not very usable in industrial applications.

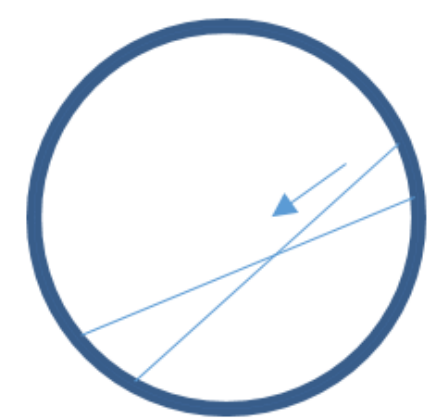

Figure 4. The slumping mode.

\subsubsection{Rolling}

When $F r$ increases to the range $0.001-1$ and $H / R$ is in the range $0.05-0.35$, the rolling mode can be observed.

In the rolling mode, particles close to the cylindrical wall moves with the wall to a certain elevation and then roll back on the surface of the bed. As a result, a circular bed movement can be observed in the rolling mode, see Figure 5. Due to this circular particle motion, most of the particles touch the cylinder bottom and the upper surface during operation, meaning that the active layer is dominant. Subsequently, the transfer of heat and mass between particles and between gas and particles is increased.

For typical industrial operations like that of a cement kiln, the rolling mode is used $[1,5]$. According to the Nielsen, et al. [5] a cement kiln is operated at 3-4 rpm.

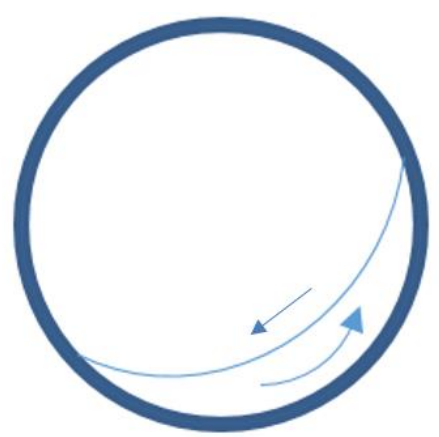

Figure 5. Rolling mode.

\subsubsection{Cascading}

When the degree of filling increases to a certain level above that of the rolling mode, the cascading mode can be seen $[11,13]$. In this mode, the active and passive layers are difficult to distinguish from each other.

In the cascading mode, the particles show a stronger accumulation on the elevated side, and the top layer of the particle bed moves more compared to the rolling mode, see Figure 6 . The cascading mode actually gives maximum mixing and maximum mass and heat transfer. However, since this operation mode consumes extra energy, it is usually not considered the optimal solution. 


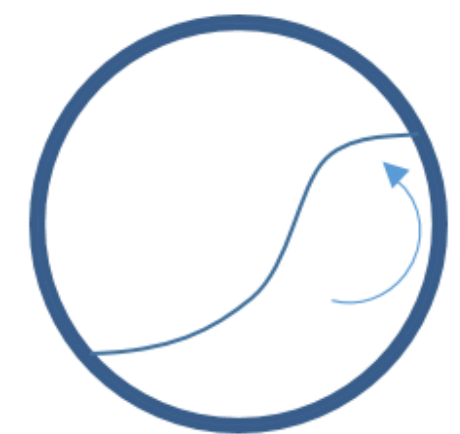

Figure 6. The cascading mode.

\subsubsection{Cataracting}

For $F r>1$ and high $H / R$ values, the cataracting mode occurs $[7,11]$, see Figure 7 . The cataracting mode can be seen in RCs operating at high rotational speeds. The cataracting mode has free falling particles at the elevated end of the cylinder, see Figure 7. Such a flow pattern gives improved mixing with gas phase inside the cylinder. However, the cataracting mode is not be considered a viable option in the industrial applications as the operational range is very narrow and the rotational speed must be very high.

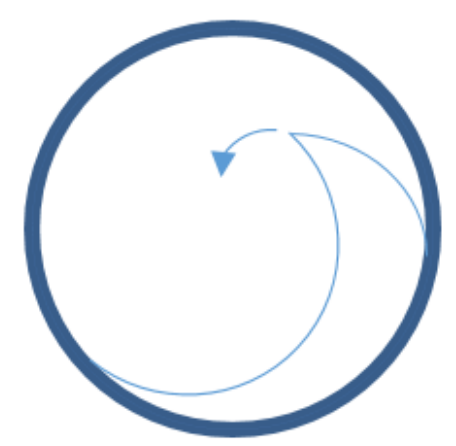

Figure 7. Cataracting mode.

\subsubsection{Centrifuging}

A further increase in $\mathrm{Fr}$ will give the centrifugal mode. In this mode, most of the particles are pressed towards the cylindrical wall due to the centrifugal force, see Figure 8. This mode requires extremely high rotational speeds. In the centrifugal mode the active layer is dominant.

For normal industrial operations, in which particle mixing and heat transfer are expected, the centrifugal mode is not used. However, it may be utilized in some applications, such as drying or separation of solids from a fluid [14].

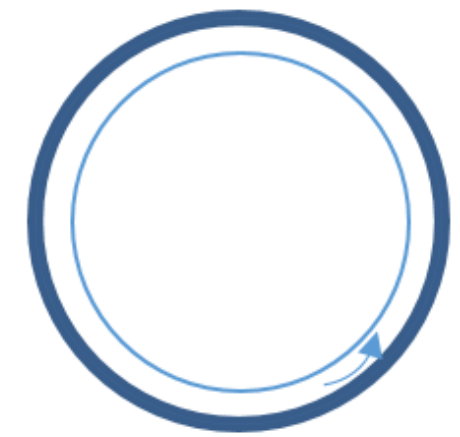

Figure 8. The centrifuging mode.

\section{Simulation study}

In this study, OpenFOAM (version 4.1) and ANSYS Fluent (release 16.2) were used, applying the EulerEuler approach, in which a set of continuity and momentum equations was solved for each phase. This means that gas and solids phases are modeled as two interpenetrating continua [2]. The method is based on property averaging in the time domain, where the local instantaneous balance of each phase is obtained. Moreover, time averaging of the fluid flow motion is applied; the Reynolds-Average Navier-Stokes (RANS) method is used to solve the properties of the turbulent flow field [15].

The details of the Eulerian model and the particle drag model, as well as additional information, are given in a previous article by some of the present authors [8, 16] and are therefore not repeated here.

The six different flow patterns illustrated in Figure 1 were simulated using the operational conditions given in Table 1.

In the simulation, a lab-scale $\mathrm{RC}$ with a radius of $0.20 \mathrm{~m}$ was considered. The diameter of full-scale cement kilns are typically 10-20 times bigger than this, but lab-scale RC has been practical to use in verification of results simulated with the Fluent software [8]. To enable comparison of OpenFOAM and Fluent results, the same RC is the basis for the current study.

In the slipping and slumping modes, the rotational speed was low $(0.67 \mathrm{rpm})$, so a relatively long operational time ( $90 \mathrm{~s}$ ) had to be simulated to make sure there was enough time for the flow pattern to develop. In the rolling and cascading modes, the rotational speed was 10 times higher $(6.67 \mathrm{rpm})$, so the time could be reduced by a factor of 10 . Moreover, in the cataracting and centrifuging modes, the rotational speeds were higher by a factor of 10 and 22.5 , respectively, so the time could be reduced accordingly. 
Table 1. Simulation parameters $(R=0.20 \mathrm{~m})$.

\begin{tabular}{|l|l|l|l|l|l|l|}
\hline Detail & Slipping & Slumping & Rolling & Cascading & Cataracting & Centrifuging \\
\hline $\begin{array}{l}\text { Degree of } \\
\text { filling, } H / R\end{array}$ & 0.10 & 0.35 & 0.20 & 0.45 & 0.45 & 0.45 \\
\hline $\begin{array}{l}\text { Rotational } \\
\text { speed }(R P M)\end{array}$ & 0.67 & 0.67 & 6.67 & 6.67 & 66.7 & 150 \\
\hline $\begin{array}{l}\text { Froude } \\
\text { number }\end{array}$ & 0.0001 & 0.0001 & 0.01 & 0.01 & 1 & 5 \\
\hline $\begin{array}{l}\text { Total time } \\
\text { simulated (s) }\end{array}$ & 90 & 90 & 9 & 9 & 0.9 & 0.4 \\
\hline
\end{tabular}

\section{Results and Discussion}

Figure 9 and 10 show the results of OpenFOAM and Fluent simulations. The red color in Figure 9 designates pure solids, whereas the blue color means no solids (i.e. gas only). Colors in between blue and red indicate areas where both phases are present. Both the Fluent and OpenFOAM simulation results match fairly well the outlined slipping mode flow pattern shown in Figure 3.
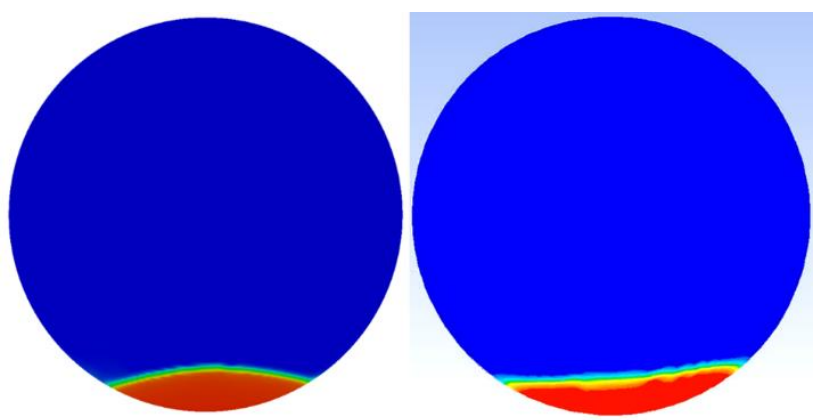

Figure 9. Slipping mode solids fraction (left: OpenFOAM, right: Fluent). Red $=100 \%$ solids, Blue $=0 \%$ solids

The OpenFOAM velocity vectors (Figure 10, left) show both the gas phase and the particle phase, while the Fluent velocity vectors (Figure 10, right) only show the particle phase (this is due to different features of the graphics processing procedures in the two software packages). The velocity vectors are qualitatively in agreement with results from previous studies [6, 12].

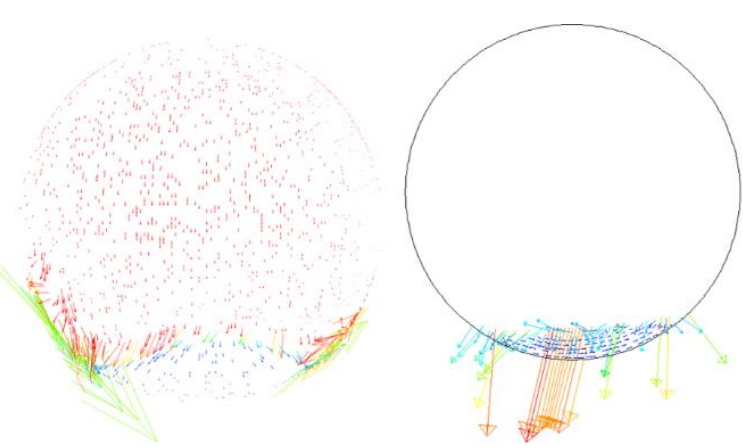

Figure 10. Slipping mode velocity vectors (left: OpenFOAM gas and particle velocities, right: Fluent particle velocities).

Figure 11 and 12 shows a comparison of the OpenFOAM and the Fluent flow patterns for the slumping mode (Figure 4), which appear after an increase in filling degree from 10 to $35 \%$. In Figure 11, the particle bed at an elevated point is shown. It is hard to capture the exact point at which the particle bed collapses.

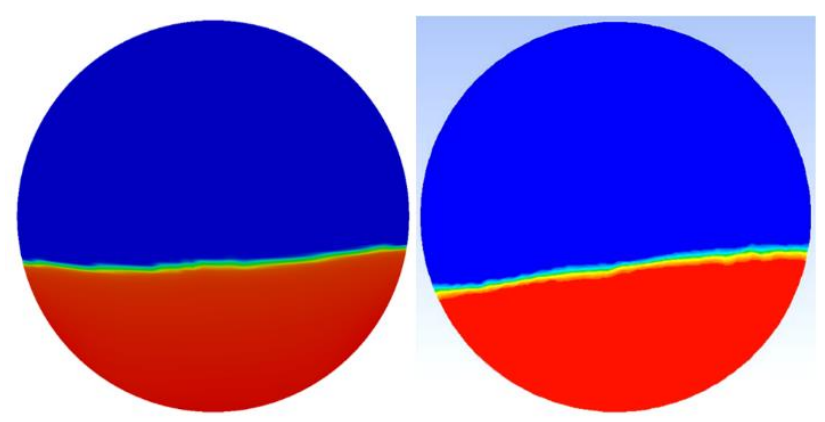

Figure 11. The slumping mode solids fraction (left: OpenFOAM, right: Fluent).

The velocity vectors are shown in Figure 12. According to the figure, the particles at the bottom move in parallel with the cylindrical wall, and the top of the bed has a backward-directed velocity. Moreover, the velocity at the top of the bed is higher than the bottom velocity. Both simulation software is capable of producing results in agreement with the theory. 


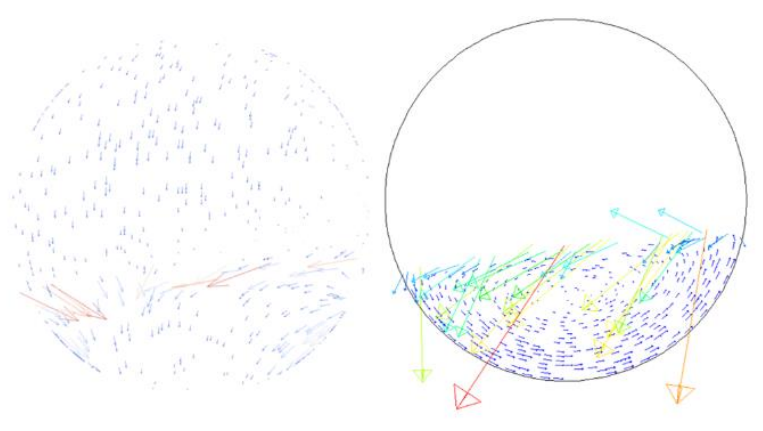

Figure 12. Slumping mode velocity vectors (left: OpenFOAM gas and particle velocities, right: Fluent particle velocities)

Figure 13 and 14 shows the rolling mode (Figure 5) results that appear after increasing the rotational speed by a factor of 10 (from 0.67 to $6.67 \mathrm{rpm}$ ) and reducing the filling degree slightly (from 35 to $20 \%$ ). The characteristics of this mode are observed both in OpenFOAM and Fluent.

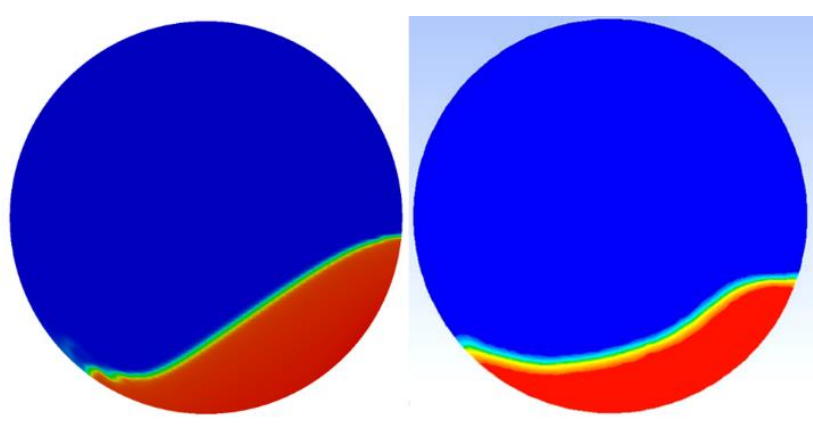

Figure 13. Rolling mode solids fraction (left: OpenFOAM right: Fluent).

The velocity vector plots in Figure 14 match each other quite well, even if the different graphics processing procedures makes the gas phase visible in OpenFOAM, but not in Fluent. The bottom particle movement and the surface particle movement are both visible from the plot, and the velocity vectors are also in line with previous studies $[6,17]$.

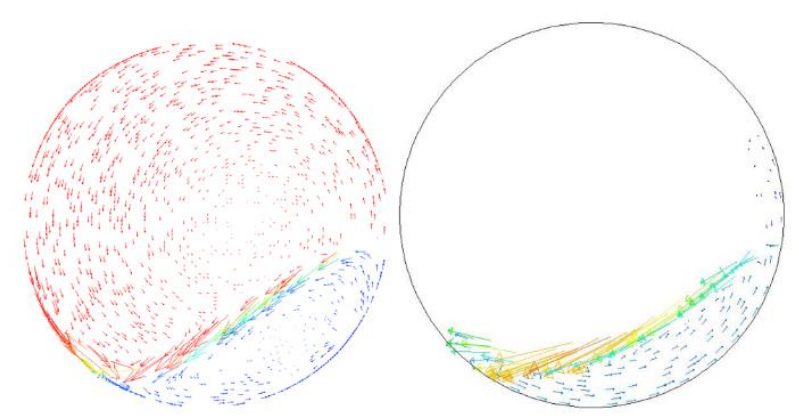

Figure 14. Rolling mode velocity vectors (left: OpenFOAM gas and particle velocities, right: Fluent particle velocities)

The cascading mode flow pattern is observed in Figure 15 and 16 . This mode was reached by increasing the filling degree from 20 to $45 \%$ (while maintaining the rotational speed at $6.67 \mathrm{rpm}$ ) and is in line with the sketch of the pattern shown in Figure 6.

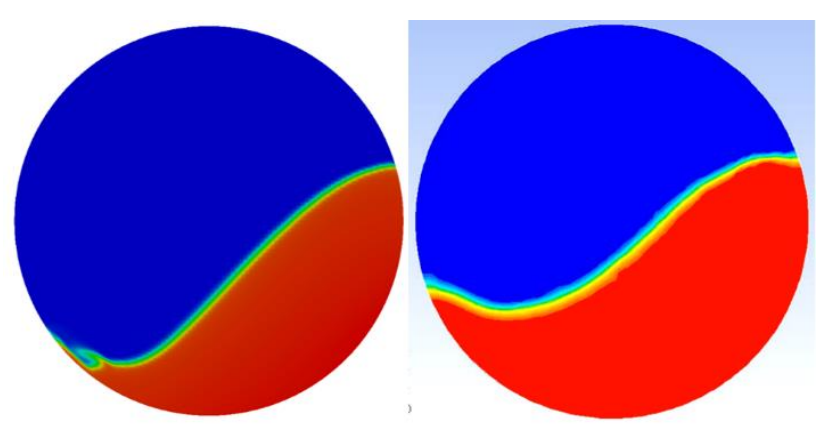

Figure 15. The cascading mode solids fraction (left: OpenFOAM, right: Fluent).

According to Figure 15, the particles accumulate more heavily on the elevated side. The particle elevation in this mode is higher than in the rolling mode.

Velocity vectors are plotted in Figure 16, and similarities can be observed. The particle velocity at the surface is higher in this mode, as the particle elevation is higher than the rolling mode.

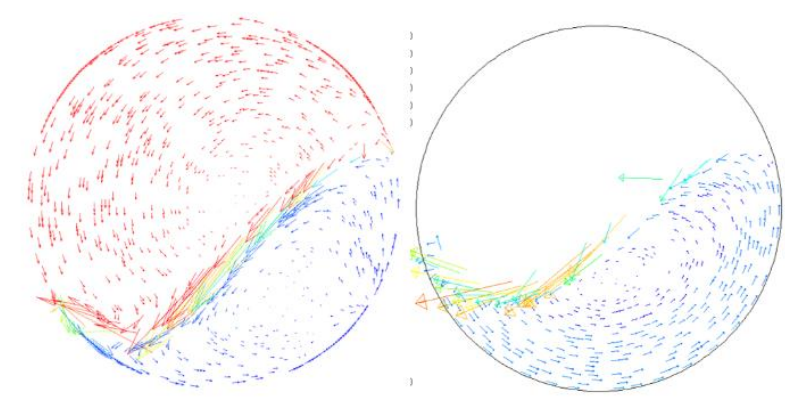

Figure 16. Cascading mode velocity vectors (left: OpenFOAM gas and particle velocities, right: Fluent particle velocities).

An increase in rotational speed by a factor of 10 (from 6.67 to 66.7 ) causes the cataracting mode (Figure 7) to appear when the filling degree is kept at $45 \%$, see Figure 17 and 18.

Figure 17 illustrates that both OpenFOAM and Fluent simulation results show particle showering down, creating a circular type of motion. The OpenFOAM contours appear to be particularly illustrative in this respect. 


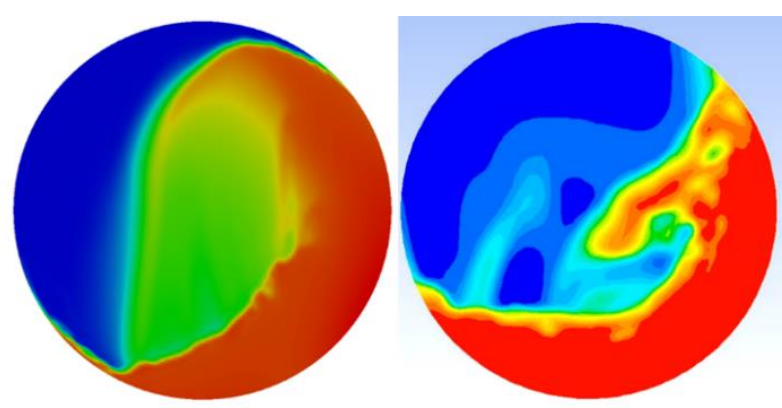

Figure 17. Cataracting mode solids fraction (left: OpenFOAM, right: Fluent).

The velocity vectors in Figure 18 match each other quite closely and also correspond well with results reported by Finnie, et al. [17].
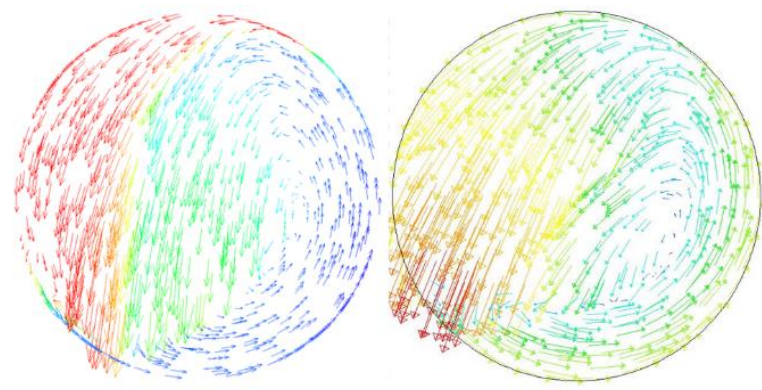

Figure 18. Cataracting mode velocity vectors (Left: OpenFOAM gas and particle velocities, right: Fluent particle velocities).

Finally, when the rotational speed is increased even more (from 66.7 to $150 \mathrm{rpm}$ ) while keeping the degree of filling unchanged $(45 \%)$, the particle bed reaches the centrifugal mode (Figure 8).

Both simulation software shows similar behavior for the solids fraction given in Figure 19.

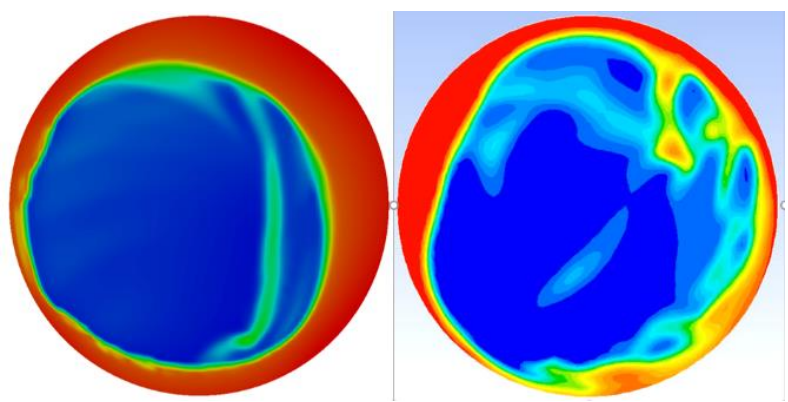

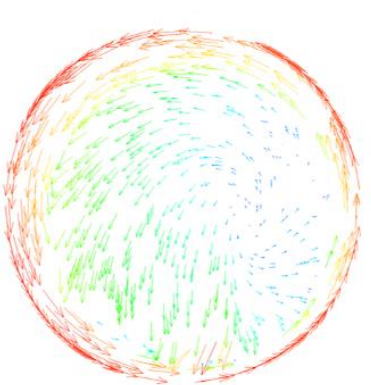

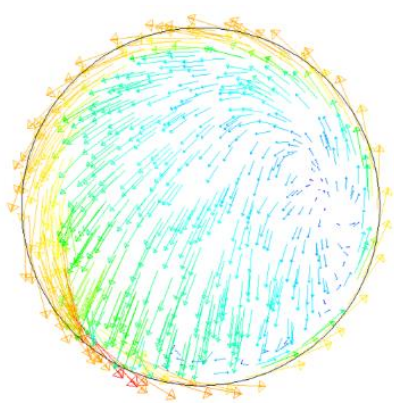

Figure 20. Centrifuging mode velocity vectors (left: OpenFOAM gas and particle velocities, right: Fluent particle velocities).

Also the velocity vector plots in Figure 20 show a close match with each other. The particle velocity at the wall has the highest velocity, and this is more or less equal to the cylindrical wall velocity.

The flow pattern development in both software for a particular case is shown in Figure 21 and Figure 22. The centrifugal mode was selected in this demonstration as it shows significant differences with time. Images were captured at $0,0.1,0.2,0.3,0.4 \mathrm{~s}$.

The flow development over time is very comparable in OpenFOAM and Fluent. At the very beginning, particles are gathered at the bottom of the cylinder at the same filling degree. As time proceeds, the particles move with the cylindrical wall, and after $0.4 \mathrm{~s}$, a more or less circular flow pattern is observed in both Fluent and OpenFOAM results.

It should be mentioned that the OpenFOAM simulations converged faster than the Fluent simulations, which can be a decisive factor when selecting what software to use. As an example, OpenFOAM converges to the solution within a day for the rolling mode, whereas the Fluent simulation takes about a week. Moreover, as the OpenFOAM is an open source software, the scripts are easily adjustable and easily manipulated by users. Contrary, the Fluent is a commercial software and the user has no access to the original scripts. The graphical interface in OpenFOAM is also very user-friendly.

Figure 19. Centrifuging mode solids fraction (left: OpenFOAM right: Fluent).
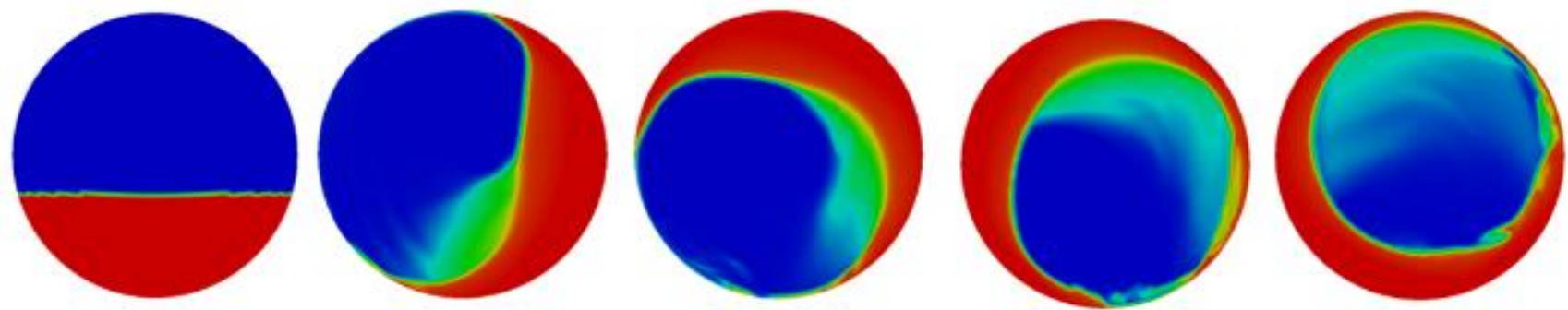

Figure 21. Flow pattern development over time (OpenFOAM); elapsed time from left to right: $0,0.1,0.2,0.3$ and 0.4 s. 

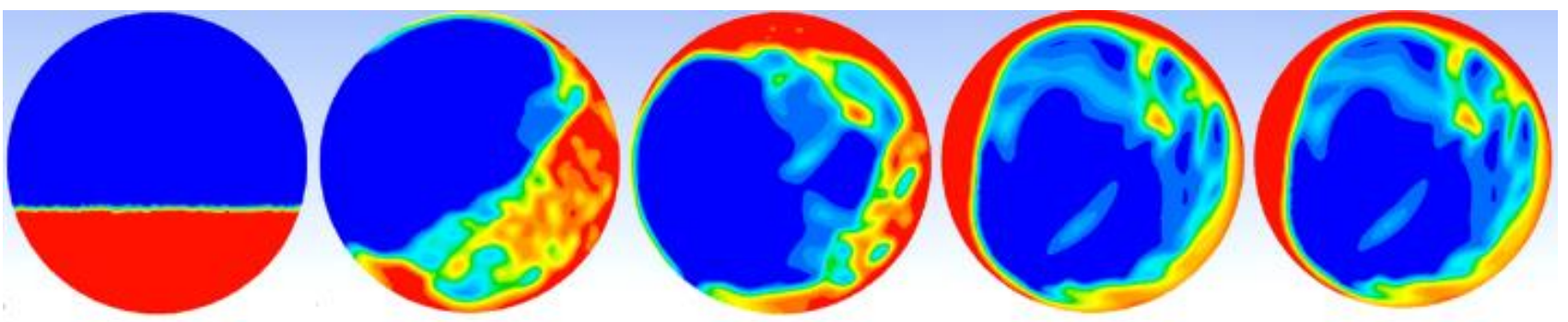

Figure 22. Flow pattern development over time (Fluent); elapsed time from left to right: $0,0.1,0.2,0.3$ and $0.4 \mathrm{~s}$.

\section{Conclusion}

Based on simulations executed with OpenFOAM and Fluent, applying the same approach and the same drag models, it can be concluded that two software packages produce very similar results. The simulated results also agreed well with flow patterns described in the literature. Hence, for a particular particle diameter, different flow regimes in an $\mathrm{RC}$ can be mapped in a diagram of filling degree vs. Froude number (which in turn depends on the rotational speed and the cylinder diameter).

OpenFOAM appears to be faster in producing the same results, and the interface is user-friendly. However, the two simulation softwares have different graphics processing procedures, which makes it difficult to generate plots that are directly comparable.

Based on the study it can be concluded that the EulerEuler approach can be successfully utilized in particlegas phase simulations and that both software platforms can be used for this purpose.

\section{References}

[1] A. A. Boateng, "4 - Granular Flows in Rotary Kilns," in Rotary Kilns, ed Burlington: ButterworthHeinemann, 2008, pp. 59-100.

[2] V. Romero, "Numerical Modelling of Granular Beds in Rotary Kilns," Master of Science in Computer Simulations for Science and Engineering Faculty of Electrical Engineering, Mathematics and Computer Science, Delft University of Technology, 2012.

[3] Y. L. Ding, J. P. K. Seville, R. Forster, and D. J. Parker, "Solids motion in rolling mode rotating drums operated at low to medium rotational speeds," Chemical Engineering Science, vol. 56, pp. 1769-1780, 3// 2001.

[4] K. S. Mujumdar and V. V. Ranade, "Simulation of Rotary Cement Kilns Using a One-Dimensional Model," Chemical Engineering Research and Design, vol. 84, pp. 165-177, 3// 2006.

[5] A. R. Nielsen, R. W. Aniol, M. B. Larsen, P. Glarborg, and K. Dam-Johansen, "Mixing large and small particles in a pilot scale rotary kiln," Powder Technology, vol. 210, pp. 273-280, 7/10/ 2011.

[6] Y. Demagh, H. Ben Moussa, M. Lachi, S. Noui, and L. Bordja, "Surface particle motions in rotating cylinders: Validation and similarity for an industrial scale kiln," Powder Technology, vol. 224, pp. 260-272, 7// 2012.

[7] M. D. Heydenrych, "Modeling of Rotary Kilns," Doctor of philosophy University of Twente, 2011.
[8] S. Karunarathna, "Investigation of Mixing and Segregation of Solids in a Rotary Kiln by CFD Simulations," Masters in Process Technology Department of Process, Energy and Environmental Technology University College of Southeast Norway

[9] H. Arastoopour, "Numerical simulation and experimental analysis of gas/solid flow systems: 1999 Fluor-Daniel Plenary lecture," Powder Technology, vol. 119, pp. 59-67, 9/24/2001.

[10] S. Benyahia, H. Arastoopour, T. M. Knowlton, and H. Massah, "Simulation of particles and gas flow behavior in the riser section of a circulating fluidized bed using the kinetic theory approach for the particulate phase," Powder Technology, vol. 112, pp. 24-33, 10/5/ 2000.

[11] Q. J. Zheng and A. B. Yu, "Modelling the granular flow in a rotating drum by the Eulerian finite element method," Powder Technology, vol. 286, pp. 361-370, $12 / / 2015$.

[12] H. Yin, M. Zhang, and H. Liu, "Numerical simulation of three-dimensional unsteady granular flows in rotary kiln," Powder Technology, vol. 253, pp. 138-145, 2// 2014.

[13] G. R. Woodle and J. M. Munro, "Particle motion and mixing in a rotary kiln," Powder Technology, vol. 76, pp. 241-245, 1993/09/01 1993.

[14] N. Barman and L. S. Badwaik, "Effect of ultrasound and centrifugal force on carambola (Averrhoa carambola L.) slices during osmotic dehydration," Ultrasonics Sonochemistry, vol. 34, pp. 37-44, 1// 2017.

[15] H. K. Versteeg and W. Malalasekera, An Introduction to Computational Fluid Dynamics, 2nd edition ed.: Pearson Education Limited, 2007.

[16] S. Karunarathna, C. Jayarathna, and L. A. Tokheim, "Impact of Particle Diameter, Particle Density and Degree of Filling on the Flow Behavior of Solid Particle Mixtures in a Rotating Drum," in The 9th Eurosim Congress on Modelling and Simulation, Oulu, Finland, 2016, pp. 955-960.

[17] G. J. Finnie, N. P. Kruyt, M. Ye, C. Zeilstra, and J. A. M. Kuipers, "Longitudinal and transverse mixing in rotary kilns: A discrete element method approach," Chemical Engineering Science, vol. 60, pp. 4083-4091, $8 / / 2005$. 